\title{
Relationship between bronchopulmonary dysplasia, long-term lung function, and vitamin D level at birth in preterm infants
}

\author{
Tiantian Lu ${ }^{1 \#}$, Bin Liang ${ }^{2 \#}$, Yanping Jia ${ }^{1}$, Jiangyun Cai ${ }^{1}$, Danhong Wang ${ }^{2}$, Min Liu ${ }^{2}$, Bo He ${ }^{2}$, Qing Wang ${ }^{3}$ \\ ${ }^{1}$ Department of Neonatology, Haikou Maternal and Child Health Hospital, Haikou, China; ${ }^{2}$ Department of Pediatric Medicine, Affiliated Haikou \\ Hospital of Xiangya Medical School Central South University, Haikou, China; ${ }^{3}$ Department of Neonatology, Hainan Modern Women and Children's \\ Hospital, Haikou, China \\ Contributions: (I) Conception and design: T Lu, B Liang, Q Wang; (II) Administrative support: Y Jia; (III) Provision of study materials or patients: \\ T Lu; (IV) Collection and assembly of data: Q Wang; (V) Data analysis and interpretation: T Lu; (VI) Manuscript writing: All authors; (VII) Final \\ approval of manuscript: All authors. \\ \#These authors contributed equally to this work. \\ Correspondence to: Qing Wang. Department of Neonatology, Hainan Modern Women and Children's Hospital, No. 18-1, Qiongzhou Avenue, \\ Qiongshan District, Haikou, China. Email: wangqing8220@163.com.
}

Background: We aimed to investigate the relationship between the level of serum 25 hydroxyvitamin D [25-(OH)D] at birth and the complications of bronchopulmonary dysplasia (BPD), as well as the long-term lung function of preterm infants.

Methods: A total of 286 premature infants who were admitted to the neonatal ward of Haikou Maternal and Child Health Hospital from January 2018 to December 2020 and met the inclusion criteria were selected as the research objects. The level of serum $25(\mathrm{OH}) \mathrm{D}$ at birth was detected. The children were divided into a BPD group (79 cases) and a non-BPD group (207 cases). The case information and basic data of the children were recorded. The children were followed up 6 months after correcting the gestational age of 40 weeks, and their long-term lung function development was reported. Logistic regression analysis was used to evaluate the high-risk factors of BPD in preterm infants.

Results: The 1- and 5-minute Apgar scores of preterm infants in the BPD group were significantly lower than those in the non-BPD group. Also, the combined neonatal pneumonia, neonatal asphyxia, hospital stay, and total oxygen therapy time in the BPD group were substantially higher than those in the non-BPD group. The mean value of serum $25-(\mathrm{OH}) \mathrm{D}$ at birth in the BPD group $(33.7 \pm 15.1 \mathrm{nmol} / \mathrm{L})$ was significantly lower than that in the non-BPD group $(49.5 \pm 19.6 \mathrm{nmol} / \mathrm{L})$. Compared with the non-BPD group, the respiratory rate $(\mathrm{RR})$ in the BPD group increased significantly, while the tidal volume (VT), inspiratory to expiratory ratio (TI/TE), ratio of time to peak tidal expiratory flow to total expiratory time (TPEF/TE), and $25 \%$ tidal expiratory flow rate (TEF25\%) decreased markedly $(\mathrm{P}<0.05)$. Total oxygen therapy time, neonatal pneumonia, neonatal asphyxia, and serum $25-(\mathrm{OH}) \mathrm{D}$ level at birth were identified as independent risk factors for BPD in preterm infants.

Conclusions: The level of serum 25-(OH)D in preterm infants at birth is closely related to the occurrence of BPD and long-term lung function damage, and is affected by multiple high-risk factors. This study provides a theoretical basis for the individualized treatment of preterm infants and the early prevention of BPD.

Keywords: Premature infants; 25 hydroxyvitamin D; bronchopulmonary dysplasia (BPD); pulmonary function

Submitted Sep 24, 2021. Accepted for publication Nov 10, 2021.

doi: $10.21037 /$ tp-21-494

View this article at: https://dx.doi.org/10.21037/tp-21-494 


\section{Introduction}

With the development of neonatal intensive care technology, the application of pulmonary surfactants, and prenatal glucocorticoids, the survival rate of preterm infants has been significantly improved. However, bronchopulmonary dysplasia (BPD) is one of the most common severe respiratory system complications in preterm infants, especially for small preterm infants. The incidence of BPD in very low birth weight infants can exceed $30 \%(1,2)$. Previous studies have shown that the occurrence of BPD can prolong the hospitalization time and increase the complications of preterm infants. In the long term, BPD can easily to lead to recurrent respiratory tract infection, wheezing, lung function damage, psychomotor retardation, and other diseases, which seriously affect the survival rate and quality of life of preterm infants (3). At present, the exact pathogenesis of BPD is still unclear, and increased levels of chemokines, inflammatory cytokines, and proteases caused by pulmonary inflammation can hinder the development and repair of neonatal lungs, and may induce BPD. There remains a lack of effective measures to prevent and treat BPD.

Vitamin D is a sterol hormone. Vitamin D deficiency can easily lead to rickets and metabolic bone diseases, as well as an increased risk of respiratory conditions such as respiratory infection (4) and bronchial asthma (5). In recent years, numerous studies have confirmed that vitamin $\mathrm{D}$ has complex biological functions, including the regulation of innate and adaptive immune function, as well as anti-inflammatory and antioxidant effects. Vitamin D also regulates lung development and maturity, and reduces lung injury caused by various factors (6-8). Studies have shown that vitamin $\mathrm{D}$ can participate in the synthesis of pulmonary surfactants, and can improve lipopolysaccharide-induced acute lung injury by maintaining the integrity of the alveolar epithelial barrier. In addition, vitamin D supplementation can significantly inhibit lung inflammation and reduce lung damage (6-8). Hence, it is related to the occurrence and development of a variety of lung diseases. In addition, a previous study also found that low serum 25-hydroxyvitamin D [25-(OH) D] level on the first day after birth was an independent risk factor for respiratory distress syndrome (7). Ge et al. reported the correlation between vitamin $\mathrm{D}$ and vitamin $\mathrm{E}$ and the occurrence of BPD, and believed that vitamin D/E deficiency was related to the severity of BPD (9). However, the correlation between vitamin $\mathrm{D}$ and long-term lung function in children with BPD is still lacking.
Therefore, this study defined the lung function of children 6 months after the correction of 40 weeks of gestational age as long-term lung function. This study aims to explore the relationship between serum 25(OH)D level at birth and BPD in preterm infants, as well as its impact on long-term lung function, so as to provide new ideas for the clinical prevention and treatment of BPD, and to improve the quality of life of children. We present the following article in accordance with the STROBE reporting checklist (available at https://dx.doi.org/10.21037/tp-21-494).

\section{Methods}

\section{Research objects}

Two hundred eighty-six premature infants who were admitted to the neonatal ward of Haikou Maternal and Child Health Hospital from January 2018 to December 2020 and met the inclusion criteria were selected as the research subjects. The inclusion criteria were as follows: (I) infants born in our hospital and transferred to our neonatal ward immediately after birth; (II) those with complete clinical case data; and (III) very low and ultra-low birth weight infants (gestational age $<37$ weeks, birth weight $<1,500 \mathrm{~g}$ preterm infants) admitted to the neonatal intensive care unit (NICU) for more than 28 days. The exclusion criteria were as follows: (I) infants with severe congenital malformations, genetic and metabolic diseases, chromosome diseases, or other organ function emergencies; (II) cases where the mother was complicated with severe liver, kidney, and thyroid diseases; (III) cases involving abandonment of treatment or death during hospitalization; and (IV) infants with incomplete personal clinical and laboratory index data. All procedures performed in this study involving human participants were in accordance with the Declaration of Helsinki (as revised in 2013). The study was approved by the Ethics Committee of Haikou Maternal and Child Health Hospital (No. 2017062) and informed consent was obtained from infants' parents.

\section{Object grouping}

Firstly, the children were divided into a BPD group (79 cases) and a non-BPD group (207 cases). Any newborn with oxygen dependence $(>21 \%)$ for more than 28 days established in the fourth edition of practical neonatology was diagnosed with BPD. Secondly, we divided the children into lung function impairment group and non-pulmonary function impairment group according to the long-term lung 
function test.

\section{Data collection and processing}

The following detailed primary clinical data of all children were collected: (I) data of preterm infants: birth gestational age, gender, weight, Apgar score, complications, days of hospitalization during hospitalization, and total oxygen therapy time; and (II) maternal data: maternal age, mode of delivery, premature rupture of membranes, preeclampsia, gestational diabetes mellitus, and prenatal hormone use.

\section{Determination of serum 25(OH)D level}

A total of $2 \mathrm{~mL}$ peripheral venous blood samples of all preterm infants and 6 months after 40 weeks of corrected gestational age included in the study were collected. After standing at room temperature for 10-20 min, they were centrifuged at $3,000 \mathrm{r} / \mathrm{min}$ at $4{ }^{\circ} \mathrm{C}$ for $15 \mathrm{~min}$ with a centrifugation radius of $13.5 \mathrm{~cm}$. After serum separation, the $25-(\mathrm{OH}) \mathrm{D}$ level was detected using the chemiluminescence analysis (CLIA) method. Detection was completed using a chemiluminescence immunoanalyzer (SIEMENS, Germany). Serum $25-(\mathrm{OH}) \mathrm{D}<50 \mathrm{nmol} / \mathrm{L}$ was diagnosed as vitamin D deficiency (10).

\section{Pulmonary function measurement}

All children included in the study were followed up, and their pulmonary function was measured 6 months after correcting the gestational age of 40 weeks. The long-term pulmonary function of preterm infants was measured by tidal respiratory pulmonary function, and its parameters can sensitively reflect the ventilation dysfunction of children with BPD. Taking the tidal volume per kilogram of body weight $(V T / \mathrm{kg})$ as the index, $V T / \mathrm{kg}<6$ was considered to indicate obstructive ventilation disorder. Other pulmonary function indexes included respiratory rate (RR), respiratory/ expiratory ratio (TI/TE), peak time ratio (TPEF/TE), peak volume ratio (VPEF/VE), and expiratory flow rate at $25 \% / 50 \% / 75 \%$ tidal volume (TEF $25 \% / 50 \% / 75 \%$ ).

\section{Statistical analysis}

SPSS 22.0 statistical software (IBM, USA) was used for the $t$-test and chi-square test. Multivariate logistic regression was used to analyze the risk factors related to BPD in children. $\mathrm{P}<0.05$ was considered statistically significant.

\section{Results}

\section{Comparison of general conditions of children}

A total of 286 eligible preterm infants were included in this study, including 79 cases in the BPD group and 207 cases in the non-BPD group. In the BPD group, there were 32 women $(40.5 \%)$. Also, the average gestational age of infants in this group was $28.9 \pm 1.7$ weeks, and the average birth weight was $1,139 \pm 143.6 \mathrm{~g}$. In the non-BPD group, there were 105 women $(50.7 \%)$, the average gestational age was $30.3 \pm 1.8$ weeks, and the average birth weight was $1,281 \pm 150.3 \mathrm{~g}$. There were no significant differences in the proportion of females, gestational age, and birth weight between the two groups $(\mathrm{P}>0.05)$. Moreover, there were no significant differences in maternal cesarean section rate, premature rupture of membranes, preeclampsia, gestational diabetes mellitus, and antenatal hormone ratio between the two groups $(\mathrm{P}>0.05)$. The 1- and 5-minute Apgar scores of preterm infants in the BPD group were markedly lower than those in the non-BPD group. Also, the combined neonatal pneumonia, neonatal asphyxia, hospital stay, and total oxygen therapy time in the BPD group were substantially higher than those in the non-BPD group $(\mathrm{P}<0.05$, Tables 1,2).

\section{Comparison of serum 25-(OH)D levels between the two groups}

The percentage of vitamin D deficiency at birth was $88.7 \%$ (70/79) in the BPD group, which was significantly higher than that of the non-BPD group $(70.5 \%, 146 / 207)$, and the difference was statistically significant $(\mathrm{P}<0.05)$. The mean values of serum $25-(\mathrm{OH}) \mathrm{D}$ at birth were $33.7 \pm 15.1$ and $49.5 \pm 19.6 \mathrm{nmol} / \mathrm{L}$ in the BPD and non-BPD groups, respectively. At 6 months after 40 weeks of corrected gestational age, the vitamin $\mathrm{D}$ deficiency rate of children in the BPD group was $72.1 \%$ (57/79), which was significantly higher than the vitamin $\mathrm{D}$ deficiency rate of children in the non-BPD group $(43.0 \%, 89 / 207)$ and the difference was statistically significant $(\mathrm{P}<0.05)$. The average serum $25-(\mathrm{OH}) \mathrm{D}$ of children in the BPD group and non-BPD group were $51.9 \pm 16.5$ and $66.3 \pm 22.1 \mathrm{nmol} / \mathrm{L}$, respectively. The difference between the two groups was statistically significant $(\mathrm{P}<0.05)$ (Table 3 and Figure 1).

\section{Long-term pulmonary function comparison between the two groups}

On pulmonary function tests, we found that long-term infants in the BPD group had significantly increased RR 
Table 1 Comparison of clinical characteristic between premature infants in the BPD and non-BPD groups

\begin{tabular}{|c|c|c|c|c|}
\hline Relevant factors & BPD group $(n=79)$ & Non-BPD group $(\mathrm{n}=207)$ & $t / \chi^{2}$ & $P$ value \\
\hline \multicolumn{5}{|l|}{ Apgar scores ( $\bar{x} \pm \mathrm{s}$, scores) } \\
\hline $1 \mathrm{~min}$ & $6.4 \pm 1.1$ & $7.9 \pm 1.1$ & $t=4.312$ & $<0.001$ \\
\hline $5 \mathrm{~min}$ & $8.1 \pm 1.2$ & $9.1 \pm 1.0$ & $\mathrm{t}=2.998$ & $<0.001$ \\
\hline Hospital stay $(x \pm s$, days $)$ & $40.3 \pm 13.1$ & $35.2 \pm 8.8$ & $t=-2.437$ & 0.032 \\
\hline Neonatal pneumonia, n (\%) & $39(49.4)$ & $46(22.2)$ & $\chi^{2}=0.096$ & $<0.001$ \\
\hline Neonatal asphyxia, n (\%) & $23(29.1)$ & $25(12.1)$ & $\chi^{2}=0.136$ & $<0.001$ \\
\hline
\end{tabular}

$\mathrm{P}<0.05$ was considered statistically significant. BPD, bronchopulmonary dysplasia.

Table 2 Comparison of maternal data and pregnancy complications between the two groups

\begin{tabular}{lcccc}
\hline Relevant factors & BPD group $(\mathrm{n}=79)$ & Non-BPD group $(\mathrm{n}=207)$ & $\chi^{2}$ & P value \\
\hline Cesarean section, $\mathrm{n}(\%)$ & $39(49.4)$ & $87(42.0)$ & 0.887 & 0.368 \\
Premature rupture of membranes, $\mathrm{n}(\%)$ & $22(27.8)$ & $59(28.5)$ & 1.017 & 0.695 \\
Preeclampsia, $\mathrm{n}(\%)$ & $8(10.1)$ & $19(9.2)$ & $36(17.4)$ & 0.968 \\
Gestational diabetes mellitus, $\mathrm{n}(\%)$ & $20(25.3)$ & $119(57.5)$ & 1.009 & 0.0677 \\
Prenatal hormone use, $\mathrm{n}(\%)$ & $43(54.4)$ & 0.846 & \\
\hline
\end{tabular}

$\mathrm{P}<0.05$ was considered statistically significant. BPD, bronchopulmonary dysplasia.

Table 3 Comparison of serum 25-(OH)D level and vitamin D deficiency rate between the two groups

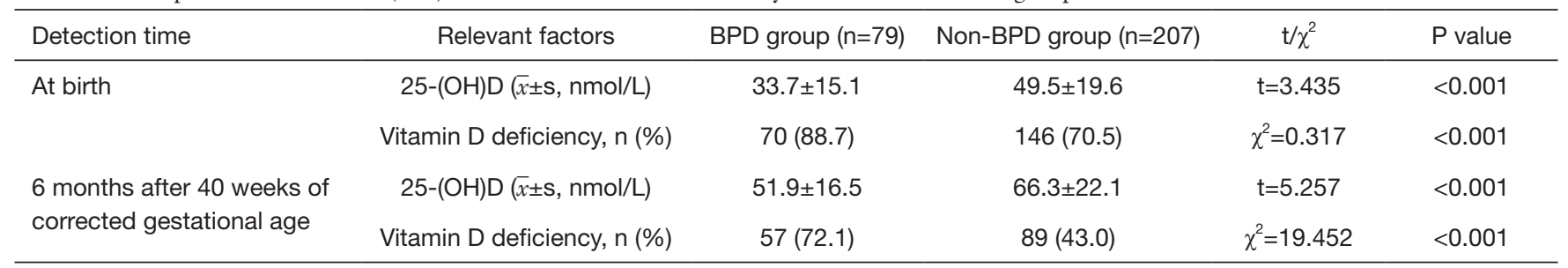

$\mathrm{P}<0.05$ was statistically significant. BPD, bronchopulmonary dysplasia.
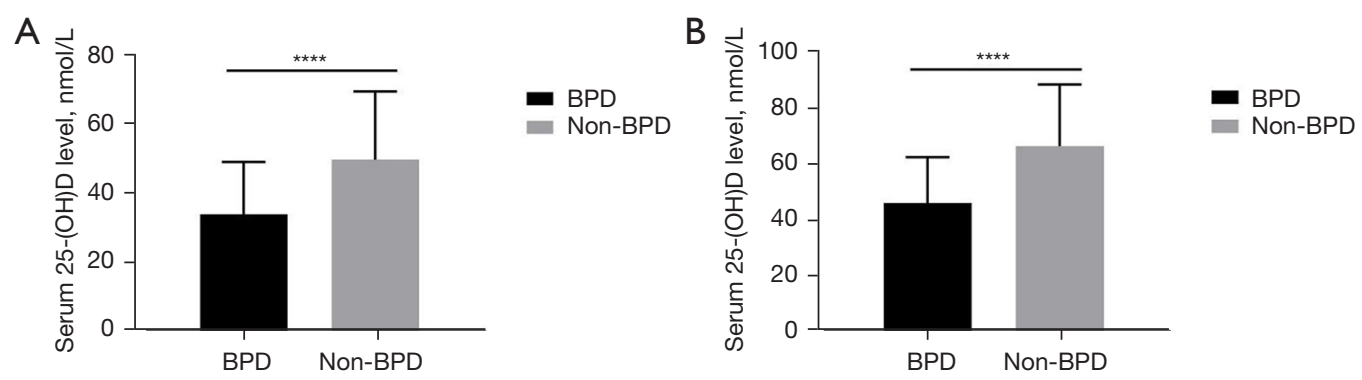

Figure 1 Comparison of serum 25-(OH)D levels between the BPD and non-BPD groups. (A) Serum 25-(OH)D level at birth; (B) serum 25-(OH)D level at 6 months after 40 weeks of corrected gestational age. ${ }^{* * *}, \mathrm{P}<0.0001$. BPD, bronchopulmonary dysplasia. 
Table 4 Comparison of the long-term lung function between the BPD and non-BPD groups

\begin{tabular}{|c|c|c|c|c|}
\hline Relevant factors & BPD group $(n=79)$ & Non-BPD group $(n=207)$ & $\mathrm{t} / \chi^{2}$ & $P$ value \\
\hline VT $(x \pm \mathrm{s}, \mathrm{mL} / \mathrm{kg})$ & $7.2 \pm 0.7$ & $8.5 \pm 0.6$ & $\mathrm{t}=4.387$ & $<0.001$ \\
\hline $\mathrm{TI} / \mathrm{TE}(x \pm \mathrm{S}, \%)$ & $72.5 \pm 7.0$ & $77.2 \pm 8.3$ & $t=3.577$ & $<0.001$ \\
\hline TPEF/TE $(x \pm \mathrm{s}, \%)$ & $29.4 \pm 4.2$ & $33.1 \pm 5.6$ & $\mathrm{t}=2.611$ & 0.004 \\
\hline TEF $25 \%(x \pm \mathrm{s}, \mathrm{mL} / \mathrm{s})$ & $53.1 \pm 7.1$ & $57.2 \pm 7.8$ & $\mathrm{t}=3.812$ & $<0.001$ \\
\hline TEF $50 \%(x \pm \mathrm{s}, \mathrm{mL} / \mathrm{s})$ & $66.5 \pm 10.5$ & $68.8 \pm 11.3$ & $\mathrm{t}=1.131$ & 0.077 \\
\hline TEF $75 \%(x \pm s, \mathrm{~mL} / \mathrm{s})$ & $71.3 \pm 12.2$ & $72.8 \pm 14.9$ & $t=1.056$ & 0.236 \\
\hline Impairment of lung function $(x, \%)$ & $63(79.7)$ & $62(29.9)$ & $\chi^{2}=57.62$ & $<0.001$ \\
\hline
\end{tabular}

$\mathrm{P}<0.05$ was considered statistically significant. $\mathrm{BPD}$, bronchopulmonary dysplasia.

Table 5 Multivariable logistic regression analysis of BPD

\begin{tabular}{lccc}
\hline \multirow{2}{*}{ Relevant factors } & \multicolumn{3}{c}{ Multivariate logistic analysis } \\
\cline { 2 - 4 } & OR & $95 \% \mathrm{Cl}$ & $\mathrm{P}$ \\
\hline Length of hospital stay & 1.08 & $0.92-1.32$ & 0.068 \\
Total oxygen therapy time & 1.33 & $1.07-3.59$ & 0.003 \\
Neonatal pneumonia & 1.25 & $1.09-1.77$ & 0.022 \\
Neonatal asphyxia & 2.33 & $1.08-4.38$ & 0.029 \\
Serum 25-(OH)D levels & 1.46 & $1.12-1.89$ & 0.013 \\
\hline
\end{tabular}

$\mathrm{P}<0.05$ was considered statistically significant. BPD, bronchopulmonary dysplasia. OR, odds ratio; $\mathrm{Cl}$, confidence interval.

and considerably lower VT, TI/TE, TPEF/TE, and TEF $25 \%$ compared to those in the non-BPD group, and the differences were statistically significant $(\mathrm{P}<0.05)$. There were no notable differences between the two groups in terms of VPEF/VE, TEF $50 \%$, and TEF $75 \%$. The proportion of children with lung function impairment in the BPD group was $79.7 \%$ (63/79), which was significantly higher than the proportion of children with lung function impairment in the non-BPD group $(29.9 \%, 62 / 207)$. The difference was statistically significant $(\mathrm{P}<0.05)$ (Table 4).

\section{Logistic regression analysis of risk factors for the development of BPD in preterm infants}

Univariate regression analysis was first performed to determine whether BPD occurred as a dependent variable in logistic regression analysis. Independent variable indicators with statistical significance $(\mathrm{P}<0.05)$ in the analysis results were included in the multivariate analysis. Finally, the length of hospital stay, total oxygen therapy duration, neonatal pneumonia, neonatal asphyxia, and serum 25$(\mathrm{OH}) \mathrm{D}$ level at birth were identified as independent variables in the multivariate logistic regression analysis. The results showed that total oxygen therapy duration, neonatal pneumonia, neonatal asphyxia, and serum 25-(OH)D levels at birth were independent risk factors for the development of BPD in preterm infants $(\mathrm{P}<0.05$, Table 5$)$.

\section{Discussion}

Although BPD is one of the most common severe complications in preterm infants, its exact pathogenesis remains unclear at present. Children with BPD can present clinically with progressive dyspnea, hypoxemia, etc. Severe cases can develop complications such as pulmonary hypertension and even pulmonary heart disease, primarily due to the pathophysiological changes secondary to the severe lung tissue damage of the affected children, including decreased pulmonary compliance and increased airway resistance dysregulated ventilation/flow ratio. Children with BPD have a poor long-term prognosis. They are prone to recurrent respiratory infections, wheezing, cardiopulmonary impairment, and backward physical development, seriously affecting their quality of life. Recent studies have found that vitamin D has complex biological functions, including the regulation of immune function, anti-inflammation, antioxidation, regulation of lung development and maturation, and alleviation of lung 
injury due to various causes. It has also been shown to be closely associated with respiratory infection (11), neonatal respiratory distress syndrome (7), chronic obstructive pulmonary disease (12), and other lung diseases. Therefore, this study aimed to identify the high-risk factors affecting the occurrence of BPD in preterm infants by exploring the relationship between serum $25-(\mathrm{OH}) \mathrm{D}$ in preterm infants and the occurrence of BPD and long-term pulmonary function impairment, in the hope of providing new ideas for the early prevention and clinical prevention of BPD.

Vitamin D levels in newborns depend mainly on maternal vitamin D levels and placental transport. Since placental transport of vitamin D occurs primarily in the third trimester, preterm infants can develop insufficient vitamin $\mathrm{D}$ reserves. At the same time, preterm infants are highly susceptible to vitamin D deficiency (13) because their intestinal flora is not established, and their intestinal absorptive function is poor. The incidence of vitamin $\mathrm{D}$ deficiency in preterm infants in this study $(75.5 \%)$ is generally consistent with the results reported in previous studies $(14,15)$. At the same time, we found that the mean serum $25-(\mathrm{OH}) \mathrm{D}$ of the preterm born BPD group in this study cohort was significantly lower than that of the non-BPD group, and a significantly higher proportion of long-term lung function abnormalities in the BPD group compared to those in the non-BPD group. The above results suggest that vitamin $\mathrm{D}$ deficiency at birth may be associated with the occurrence of BPD and long-term lung function impairment.

In recent years, there have been relevant studies exploring the correlation between serum 25-(OH)D levels and the occurrence of BPD in preterm infants. Çetinkaya et al. (16) found that serum $25-(\mathrm{OH}) \mathrm{D}$ levels at birth were significantly lower in preterm infants in the BPD group than in the nonBPD group in a study of 132 preterm infants $\leq 32$ weeks gestational age. Mao et al. (17) reported similar conclusions and indicated that the $25-(\mathrm{OH}) \mathrm{D}$ level is an important factor in predicting BPD occurrence. Vitamin D may exert biological functions by acting via the vitamin $\mathrm{D}$ receptor on vitamin D pathway genes, many of which regulate lung development (18). Vitamin D may also play a key role in lung maturation by stimulating lung epithelial-mesenchymal cell interactions (19). In addition, studies have found that vitamin $\mathrm{D}$ also reduces the synthesis of transforming growth factor- $\beta$ (TGF- $\beta$ ), matrix metalloprotein (MMP), and tumor necrosis factor $(\mathrm{TNF}-\alpha)$ to inhibit airway remodeling $(16,17)$, and can attenuate lung injury caused by hyperoxia (12). The above mechanisms may collectively constitute a relevant link through which vitamin D influences the occurrence of
BPD. In the present study, preterm infants in the BPD group had lower serum 25-(OH)D levels at birth than those in the non-BPD group, and serum $25-(\mathrm{OH}) \mathrm{D}$ levels at birth were independent risk factors for the development of BPD. At the same time, At the same time, the long-term lung function damage of children in the BPD group was significantly more than that in the non-BPD group, , which also suggests that the serum $25-(\mathrm{OH}) \mathrm{D}$ level at birth and the occurrence of BPD in preterm infants have a suggestive role in longterm lung function impairment. Therefore, in order to prevent the occurrence of neonatal BPD and long-term lung function damage, regular pregnancy checkups can be taken for pregnant women to prevent premature delivery. Active treatment of infections and inflammatory diseases in pregnant women can avoid causing mother and infant inflammatory reactions. Timely supplementation of vitamin $\mathrm{D}$ intake can promote fetal lung maturity and other measures.

In conclusion, the present study suggests that serum 25$(\mathrm{OH}) \mathrm{D}$ levels at birth in preterm infants may be involved in regulating the occurrence and development of BPD and can affect the long-term pulmonary function situation in the affected infants. This study provides a theoretical basis for the early prevention and individualized treatment of BPD and long-term lung function impairment in preterm infants.

\section{Acknowledgments}

Funding: The project was supported by the Hainan Natural Science Foundation (No. 819QN388).

\section{Footnote}

Reporting Checklist: The authors have completed the STROBE reporting checklist. Available at https://dx.doi. org/10.21037/tp-21-494

Data Sharing Statement: Available at https://dx.doi. org/10.21037/tp-21-494

Conflicts of Interest: All authors have completed the ICMJE uniform disclosure form (available at https://dx.doi. org/10.21037/tp-21-494). The authors have no conflicts of interest to declare.

Ethical Statement: The authors are accountable for all aspects of the work in ensuring that questions related to the accuracy or integrity of any part of the work are appropriately investigated and resolved. All procedures 
performed in this study involving human participants were in accordance with the Declaration of Helsinki (as revised in 2013). The study was approved by the Ethics Committee of Haikou Maternal and Child Health Hospital (No. 2017062) and informed consent was obtained from infants' parents.

Open Access Statement: This is an Open Access article distributed in accordance with the Creative Commons Attribution-NonCommercial-NoDerivs 4.0 International License (CC BY-NC-ND 4.0), which permits the noncommercial replication and distribution of the article with the strict proviso that no changes or edits are made and the original work is properly cited (including links to both the formal publication through the relevant DOI and the license). See: https://creativecommons.org/licenses/by-nc-nd/4.0/.

\section{References}

1. Patel RM. Short- and Long-Term Outcomes for Extremely Preterm Infants. Am J Perinatol 2016;33:318-28.

2. Stoll BJ, Hansen NI, Bell EF, et al. Trends in Care Practices, Morbidity, and Mortality of Extremely Preterm Neonates, 1993-2012. JAMA 2015;314:1039-51.

3. Islam JY, Keller RL, Aschner JL, et al. Understanding the Short- and Long-Term Respiratory Outcomes of Prematurity and Bronchopulmonary Dysplasia. Am J Respir Crit Care Med 2015;192:134-56.

4. Dinlen N, Zenciroglu A, Beken S, et al. Association of vitamin $\mathrm{D}$ deficiency with acute lower respiratory tract infections in newborns. J Matern Fetal Neonatal Med 2016;29:928-32.

5. Chawes BL, Bønnelykke K, Jensen PF, et al. Cord blood 25(OH)-vitamin D deficiency and childhood asthma, allergy and eczema: the COPSAC2000 birth cohort study. PLoS One 2014;9:e99856.

6. Gil Á, Plaza-Diaz J, Mesa MD. Vitamin D: Classic and Novel Actions. Ann Nutr Metab 2018;72:87-95.

7. Mohamed Hegazy A, Mohamed Shinkar D, Refaat Mohamed N, et al. Association between serum $25(\mathrm{OH})$ vitamin $\mathrm{D}$ level at birth and respiratory morbidities among preterm neonates. J Matern Fetal Neonatal Med 2018;31:2649-55.

8. Lykkedegn S, Sorensen GL, Beck-Nielsen SS, et al. The impact of vitamin $\mathrm{D}$ on fetal and neonatal lung maturation. A systematic review. Am J Physiol Lung Cell Mol Physiol 2015;308:L587-602.

9. Ge H, Liu $\mathrm{W}, \mathrm{Li} \mathrm{H}$, et al. The association of vitamin D and vitamin $\mathrm{E}$ levels at birth with bronchopulmonary dysplasia in preterm infants. Pediatr Pulmonol 2021;56:2108-13.

10. Holick MF, Binkley NC, Bischoff-Ferrari HA, et al. Evaluation, treatment, and prevention of vitamin D deficiency: an Endocrine Society clinical practice guideline. J Clin Endocrinol Metab 2011;96:1911-30.

11. Mandell E, Seedorf G, Gien J, et al. Vitamin D treatment improves survival and infant lung structure after intraamniotic endotoxin exposure in rats: potential role for the prevention of bronchopulmonary dysplasia. Am J Physiol Lung Cell Mol Physiol 2014;306:L420-8.

12. Kose M, Bastug O, Sonmez MF, et al. Protective effect of vitamin $\mathrm{D}$ against hyperoxia-induced lung injury in newborn rats. Pediatr Pulmonol 2017;52:69-76.

13. Rodda CP, Benson JE, Vincent AJ, et al. Maternal vitamin $\mathrm{D}$ supplementation during pregnancy prevents vitamin $\mathrm{D}$ deficiency in the newborn: an open-label randomized controlled trial. Clin Endocrinol (Oxf) 2015;83:363-8.

14. Monangi N, Slaughter JL, Dawodu A, et al. Vitamin D status of early preterm infants and the effects of vitamin D intake during hospital stay. Arch Dis Child Fetal Neonatal Ed 2014;99:F166-8.

15. Natarajan CK, Sankar MJ, Agarwal R, et al. Trial of daily vitamin $\mathrm{D}$ supplementation in preterm infants. Pediatrics 2014;133:e628-34.

16. Çetinkaya M, Çekmez F, Erener-Ercan T, et al. Maternal/neonatal vitamin D deficiency: a risk factor for bronchopulmonary dysplasia in preterms? J Perinatol 2015;35:813-7.

17. Mao X, Qiu J, Zhao L, et al. Vitamin D and IL-10 Deficiency in Preterm Neonates With Bronchopulmonary Dysplasia. Front Pediatr 2018;6:246.

18. Chen L, Wilson R, Bennett E, et al. Identification of vitamin $\mathrm{D}$ sensitive pathways during lung development. Respir Res 2016;17:47.

19. Saadoon A, Ambalavanan N, Zinn K, et al. Effect of Prenatal versus Postnatal Vitamin D Deficiency on Pulmonary Structure and Function in Mice. Am J Respir Cell Mol Biol 2017;56:383-92.

(English Language Editor: A. Kassem)

Cite this article as: Lu T, Liang B, Jia Y, Cai J, Wang D, Liu M, He B, Wang Q. Relationship between bronchopulmonary dysplasia, long-term lung function, and vitamin $\mathrm{D}$ level at birth in preterm infants. Transl Pediatr 2021;10(11):3075-3081. doi: 10.21037/tp-21-494 